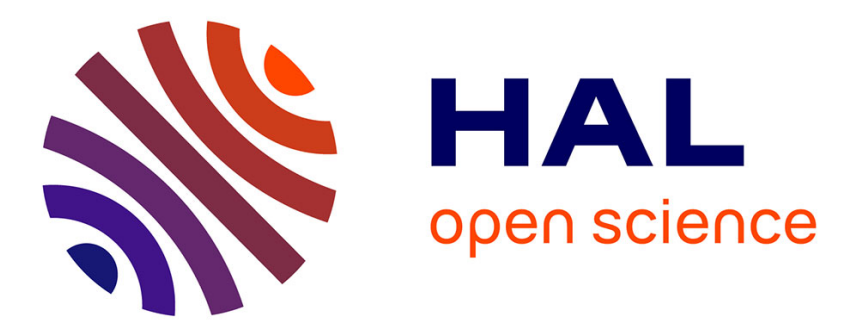

\title{
Co-Refinement of Diffraction Anomalous Fine-Structure Data
}

\author{
M. Newville, J. Cross, B. Ravel, L. Sorensen, C. Bouldin, Y. Yacoby
}

\section{To cite this version:}

M. Newville, J. Cross, B. Ravel, L. Sorensen, C. Bouldin, et al.. Co-Refinement of Diffraction Anomalous Fine-Structure Data. Journal de Physique IV Proceedings, 1997, 7 (C2), pp.C2-759-C2-760. 10.1051/jp4:1997228 . jpa-00255307

\section{HAL Id: jpa-00255307 https://hal.science/jpa-00255307}

Submitted on 1 Jan 1997

HAL is a multi-disciplinary open access archive for the deposit and dissemination of scientific research documents, whether they are published or not. The documents may come from teaching and research institutions in France or abroad, or from public or private research centers.
L'archive ouverte pluridisciplinaire HAL, est destinée au dépôt et à la diffusion de documents scientifiques de niveau recherche, publiés ou non, émanant des établissements d'enseignement et de recherche français ou étrangers, des laboratoires publics ou privés. 


\title{
Co-Refinement of Diffraction Anomalous Fine-Structure Data
}

\author{
M. Newville, J.O. Cross*, B. Ravel**, L.B. Sorensen**, C.E. Bouldin*** and Y. Yacoby**** \\ Lawrence Livermore National Laboratory, L-219, Livermore CA 94550, U.S.A. \\ * Naval Research Laboratory, Code 6680, Washington DC 20375, U.S.A. \\ ** Department of Physics, Box 351560, University of Washington, Seattle, WA 98195, U.S.A. \\ *** National Institute of Standards and Technology, Gaithersburg MD 20899, U.S.A. \\ **** Racah Institute of Physics, Hebrew University, Jerusalem, Israel
}

\begin{abstract}
Diffraction Anomalous Fine-Structure (DAFS) combines the sensitivity to long-range-order of diffraction with the short-range-order sensitivity of XAFS. This makes it possible to use a set of DAFS measured intensities to simultaneously refine both long- and short-range structural parameters, while maintaining some constraints between them. This method combines a calculation of the structure factor based on the unit cell of the crystal with a calculation of the fine-structure $\chi(E)$ around each resonant site. Tabulated values of the scattering amplitude are used away from the resonant energies, while the near-edge anomalous scattering amplitude is calculated for the resonant sites using a differential Kramers-Krönig transform of an embedded atom absorption coefficient $\mu_{0}(E)$ from FEFF. We discuss some of the subtleties of this approach to DAFS analysis.
\end{abstract}

\section{INTRODUCTION}

Diffraction anomalous fine-structure (DAFS)[1] depends on both the long-range order of the crystalline sample, and on the near-neighbor bonds. In this way, it combines the sensitivities of $x$-ray diffraction (XRD) and x-ray-absorption fine-structure (XAFS). Since these long- and short-range order are different measures of the atomic arrangement of the crystal, DAFS spectra can be used to simultaneously refine both crystallographic and near-neighbor bonding parameters. The ability to model and interpret DAFS provides an opportunity to give a more complete understanding of atomic configurations of crystals than can be found by either XRD or XAFS alone.

In order to retain the long-range order sensitivity of the DAFS spectra, the energy-dependent measured diffracted intensities themselves must be used, and the spectra should not be reduced to XAFS-like fine-structure before analysis. This presents a few new challenges to understanding DAFS, but also allows a more general analysis of DAFS spectra. One advantage of analyzing the measured energy-dependent diffracted intensity is that non-centrosymmetric crystals can be analyzed, which is not possible when using the iterative Kramers-Krönig method.[2, 3] In this paper, we emphasize the use of DAFS to get a consistent picture of the atomic configuration by establishing constraints between the long- and short-range structural parameters.

\section{ATOMIC SCATTERING FACTORS}

In the kinematic approximation, the energy-dependent diffracted intensity is proportional to the squared magnitude of the structure factor,

$$
I(\mathbf{Q}, E) \propto|F|^{2}=\left|\sum_{n} f_{n}(\mathbf{Q}, E) e^{i \mathbf{Q} \cdot \mathbf{R}_{n}} e^{-M_{n}}\right|^{2},
$$

where $\mathbf{R}_{n}$ is the position of the $n^{\text {th }}$ atom in the unit cell, $\mathbf{Q}$ is the diffraction wavevector, $E$ is the $\mathrm{X}$-ray energy, and $e^{M_{n}}$ is the crystallographic Debye-Waller factor. The atomic x-ray scattering amplitude, $f_{n}(\mathbf{Q}, E)$, is given by

$$
f_{n}(\mathbf{Q}, E)=f_{0}(\mathbf{Q})+f_{\mathrm{a}}^{\prime}(E)+i f_{\mathrm{a}}^{\prime \prime}(E)+f_{\mathbf{r}}^{\prime \prime}(E) \tilde{\chi}(E),
$$

where $f_{0}(\mathbf{Q})$ is the Thomson scattering amplitude, and $\Delta f_{\mathrm{a}}(E)=f_{\mathrm{a}}^{\prime}(E)+i f_{\mathrm{a}}^{\prime \prime}(E)$ is the anomalous scattering amplitude without any fine-structure. The fine-structure $\tilde{\chi}(E)$ is due to the backscattering of the virtual photo-electron from the neighboring atoms, and is analogous to the XAFS. $f_{\mathrm{r}}^{\prime \prime}(E)$ is the resonant anomalous scattering amplitude due to the resonant core level alone. For non-resonant atoms, $\tilde{\chi}(E)$ is negligible, and $f_{n}(\mathbf{Q}, E)=f_{0}(\mathbf{Q})+\Delta f_{\mathbf{a}}(E)$.

To model the DAFS intensity, accurate values for the anomalous scattering factors $f_{\mathrm{a}}^{\prime}(E), f_{\mathrm{a}}^{\prime \prime}(E)$, and $f_{\mathrm{r}}^{\prime \prime}(E)$ are needed near the resonance (or absorption edge) energy. While these functions are tabulated[4] for anomalous x-ray scattering work, they are not accurate enough near the absorption edge energy to be reliable for DAFS analysis. To overcome this problem, we use FEFF calculations for $\mu_{0}(E)$, which is simply related to $f_{\mathrm{r}}^{\prime \prime}(E)$, and can easily be made to improve both $f_{\mathrm{r}}^{\prime \prime}(E)$ and $f_{\mathrm{a}}^{\prime \prime}(E)$ enough to be useful in DAFS analysis.

To improve $f_{a}^{\prime}(E)$, the real part of the anomalous scattering amplitude, we exploit its relation to $f_{a}^{\prime \prime}(E)$ through the Kramers-Krönig transform. Since this transform is linear, and since the tabulated $\Delta f_{\mathbf{a}}(E)$ are analytic, the Kramers- 
Krönig transform of the improvement in $f_{\mathrm{a}}^{\prime \prime}(E)$ gives the improvement in $f_{\mathrm{a}}^{\prime}(E)$.[3] In this way, a FEFF calculation of $\mu_{0}(E)$ can be used to accurately improve the anomalous scattering amplitudes used in DAFS analysis.

\section{STRUCTURAL REFINEMENT}

Once reliable values for the anomalous atomic scattering factors have been determined, refinement of the full DAFS intensity with both long- and short-range order parameters can be done. As a simple example, consider the two-atom unit cell shown in Fig. 1, with site 1 being far from resonance (and with $\Delta f_{\mathrm{a}}(E)=0$ ) and site 2 being resonant. Writing $\tilde{\chi}$ explicitly as a sum over scattering-paths, the structure factor is

$$
F=f_{0_{1}} e^{i \mathbf{Q} \cdot \mathbf{R}_{1}} e^{-M_{1}}+\left[f_{0_{2}}+f_{\mathbf{a}_{2}}^{\prime}+i f_{\mathbf{a}_{2}}^{\prime \prime}+i f_{\mathrm{r}_{2}}^{\prime \prime} \sum_{m} \frac{N_{m} \mathcal{F}_{\text {eff }}}{k R_{2 m}^{2}} e^{i\left(2 k R_{2 m}+\phi_{m}\right)} e^{-2 k^{2} \sigma_{2 m}^{2}}\right] e^{i \mathbf{Q} \cdot \mathbf{R}_{2}} e^{-M_{2}} .
$$

The fine-structure sum includes a term with photo-electron backscattering from the non-resonant atom $(m=1)$, which depends on $R_{21}=\left|\mathbf{R}_{2}-\mathbf{R}_{1}\right|$, the distance between sites 1 and 2. The DAFS intensity then depends on $\mathbf{R}_{1}$, $\mathbf{R}_{2}$, and $R_{21}$. Since these terms are obviously related, the refinement of the DAFS intensity must depend on these quantities in a self-consistent manner. Similar relations can be written for all paths in terms of atomic positions within the unit cell and unit cell parameters. Such self-consistencies makes this approach to DAFS analysis quite attractive, as the diffraction and fine-structure measurement probe different parts of the atomic configuration.

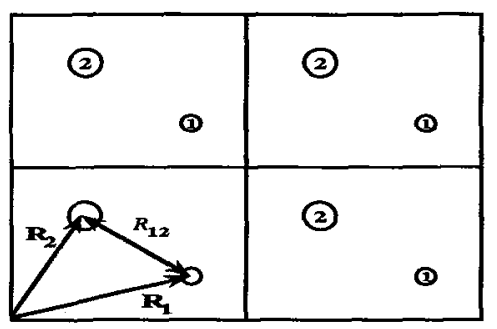

Figure 1: Schematic diagram of a two-site lattice. XRD probes $\mathbf{R}_{1}$ and $\mathbf{R}_{2}$, while XAFS probes $R_{12}$. A DAFS measurement is sensitive to all these quantities.

In addition to depending on both lattice positions and bond lengths, DAFS measurements contain two different probes of the positional and thermal disorder of a crystal. The diffraction Debye-Waller factor depends on $\left\langle u_{n}^{2}\right\rangle$, the mean-square displacement of an atom around its lattice site, while $\tilde{\chi}$ depends on $\sigma^{2}$, the mean-square displacement of the interatomic bond length. These two are related to one another and to the degree of correlation of the displacements of the two atoms. For a simple case, $\sigma_{n m}^{2}=\left\langle u_{n}^{2}\right\rangle+\left\langle u_{m}^{2}\right\rangle-2\left\langle u_{n} u_{m}\right\rangle$. A DAFS determination of $\left\langle u_{n}^{2}\right\rangle,\left\langle u_{m}^{2}\right\rangle$, and $\sigma_{n m}^{2}$ can be used to evaluate the correlation $\left\langle u_{n} u_{m}\right\rangle$ directly.

\section{CONCLUSION}

The capabilities of DAFS described here require special attention to the experimental measurements. In order to be sensitive to long-range order, a large set of diffraction intensities is needed. These can be made at a single energy, far from the resonance energy, as is done for most diffraction experiments. Energy-dependent scans through the resonant energy with energy ranges and grids similar to those of an XAFS experiment are needed at a much smaller number of $\mathbf{Q}$ values. The number of energy-dependent scans should exceed the number of unique unit-cell sites for the resonant atom. The polarization dependence of DAFS causes the short-range sensitivity to be perpendicular to the long-range sensitivity at any one reflection, so that all reflections must be refined together. The ability to simultaneously refine the full DAFS intensity provides a unique opportunity to use both the diffraction long-range order sensitivity of diffraction and the short-range order sensitivity of XAFS in a way that gives a consistent view of the atomic structure of a crystal.

\section{References}

[1] Sorensen L. B., Cross J. O., Newville M., Ravel B., Rehr J. J., Stragier H. J., Bouldin C. E. and Woicik J. C., "Diffraction anomalous fine structure: Unifying x-ray diffraction and x-ray absorption with DAFS," in Resonant Anomalous $X$-Ray Scattering: Theory and Applications, G. Materlik, C.J. Sparks and K. Fischer, Eds. (Elsevier Science B.V., 1994) pp. 389420.

[2] Pickering I. J., Sansone M., Marsch J., and George G. N., J. Am. Chem. Soc. 115 (1993) 6302-6311.

[3] Cross J. O., Ph. D. thesis, University of Washington, 1996.

[4] Sasaki S., Numerical Tables of Anomalous Scattering Factors Calculated by the Cromer and Liberman's Method, KEK Report 88-14 (National Laboratory for High Energy Physics, 1989). 